\title{
Spotlight on reticular pseudodrusen
}

This article was published in the following Dove Press journal:

Clinical Ophthalmology

20 September 2017

Number of times this article has been viewed

\author{
Alessandro Rabiolo' \\ Riccardo Sacconi ${ }^{1,2}$ \\ Maria Vittoria Cicinelli' \\ Lea Querques 1,3 \\ Francesco Bandello' \\ Giuseppe Querques' \\ 'Department of Ophthalmology, \\ University Vita-Salute, IRCCS \\ San Raffaele, Milan, ${ }^{2}$ Eye Clinic, \\ Department of Neurological and \\ Movement Sciences, University \\ of Verona, Verona, ${ }^{3} \mathrm{G}$. B. Bietti \\ Foundation-IRCCS, Rome, Italy
}

\begin{abstract}
Age-related macular degeneration (AMD) is a leading cause of vision loss in patients $>50$ years old. The hallmark of the disease is represented by the accumulation of extracellular material between retinal pigment epithelium and the inner collagenous layer of Bruch's membrane, called drusen. Although identified almost 30 years ago, reticular pseudodrusen (RPD) have been recently recognized as a distinctive phenotype. Unlike drusen, they are located in the subretinal space. RPD are strongly associated with late AMD, especially geographic atrophy, type 2 and 3 choroidal neovascularization, which, in turn, are less common in typical AMD. RPD identification is not straightforward at fundus examination, and their identification should employ at least 2 different imaging modalities. In this narrative review, we embrace all aspects of RPD, including history, epidemiology, histology, imaging, functional test, natural history and therapy.
\end{abstract}

Keywords: age-related macular degeneration, choroidal neovascularization, geographic atrophy, reticular macular degeneration, reticular drusen, reticular macular disease, reticular pseudodrusen, subretinal drusenoid deposit

\section{Introduction}

Age-related macular degeneration (AMD) is a chronic, progressive degenerative retinal disease characterized by loss of central vision and represents one of the leading causes of blindness in subjects $>50$ years old. ${ }^{1}$ Being a distinctive feature of AMD, drusen appear on fundus examination as focal yellowish dots clustered at the posterior pole and are constituted by extracellular material accumulation between retinal pigment epithelium (RPE) and the inner collagenous layer of Bruch's membrane (BM). ${ }^{1}$ In recent times, a distinctive phenotype, called reticular pseudodrusen (RPD), has been in the spotlight.

Here, we aim to provide the reader a comprehensive narrative review embracing all aspects of RPD.

\section{Literature search}

A PubMed engine search was carried out using the terms "reticular pseudodrusen", "reticular drusen", "subretinal drusenoid deposits", and "reticular macular disease". All studies published in English up to July 2017, irrespective of their publication status, were reviewed and relevant publications were included in this review.

\section{History}

Mimoun et $\mathrm{al}^{2}$ described for the first time "pseudodrusen visible en lumière bleue" in 1990 to define retinal lesions with a variable diameter of about $100 \mu \mathrm{m}$ that did not appear hyperfluorescent on fluorescein angiography (FA), but with enhanced visibility in blue light. In 1991, RPD were incorporated into the Wisconsin AMD grading system 
as "ill-defined networks of broad interlacing ribbons" on color fundus photography and were named reticular soft drusen. ${ }^{3}$ In 1995, Arnold et $\mathrm{al}^{4}$ further characterized RPD as "yellow interlacing network $125-250 \mu \mathrm{m}$ wide appearing first in the superior outer macula and then extending circumferentially and beyond" and coined the terminology RPD. Since then, the nomenclature RPD spread in the current literature, although other terms (ie, subretinal drusenoid deposits, reticular macular disease, reticular drusen) have been used.

\section{Epidemiology}

\section{Prevalence}

Prevalence of RPD varies in relation to the modality of evaluation and the cohort of patients enrolled. Prevalence of RPD in general elder population was assessed by large, long-term follow-up prospective studies, namely, Beaver Dam Eye, Blue Mountains Eye and Melbourne Collaborative Cohort studies. ${ }^{5-7}$ In those studies, the prevalence of RPD turned to be $0.41 \%-1.95 \%$ and $3.0 \%-4.0 \%$ at baseline and 15 years, respectively. ${ }^{5-7}$ However, the sensitivity of color fundus photography is very low. Conversely, novel imaging modalities (ie, spectral-domain optical coherence tomography [SDOCT], infrared [IR] reflectivity and multicolor) exhibited excellent sensitivity/specificity, and, therefore, studies employing these modalities disclosed higher prevalence of RPD. ${ }^{8}$ In the Rotterdam Eye Study, RPD were detected in about $5 \%$ of participants using color fundus photograph (CFP) and near-infrared reflectance (NIR). ${ }^{9}$ Prevalence of RPD was even higher ranging from $13.4 \%$ to $32 \%$ when multimodal imaging was employed. ${ }^{10,11}$ Interestingly, RPD seem to be less frequent in Asiatic compared with White populations. ${ }^{12}$ RPD are bilateral in $50 \%-84 \%$ of cases. ${ }^{6,13-15}$

Independently from the macular status, the presence of RPD in older population increased 3.4-fold the chance to suffer from AMD in general elder population. ${ }^{11}$ Prevalence of RPD in patients with AMD is obviously higher than that in general population, ranging from $16.8 \%$ to $79 \%$, depending on the imaging modality used, stage and type of AMD. ${ }^{8,11,16-20}$ Surprisingly, RPD prevalence was even higher $(85 \%)$ in postmortem histological samples of patients with AMD.$^{21}$ Although their overall presence in neovascular AMD ranges between $22 \%$ and $36 \%$, RPD are more easily found in eyes with type 3 choroidal neovascularization (CNV) (68.4\%-83\%). Conversely, they are less commonly seen in eyes with typical neovascular AMD (type 1 and 2) or polypoidal choroidal vasculopathy with a prevalence of 9\%-13.9\% and $2 \%-3.4 \%$, respectively. ${ }^{17,19,22,23}$ In eyes with geographic atrophy, the prevalence of RPD ranges between $29 \%$ and
50\%. ${ }^{14,17}$ An association between RPD and acquired vitelliform detachment has been reported, suggesting RPE dysfunction and impairment to outer segments turnover. ${ }^{24,25}$

Although RPD are commonly found in AMD, they have also been associated with other retinal affections, including early-onset drusen, ${ }^{26}$ pseudoxanthoma elasticum, ${ }^{27,28}$ Sorsby macular dystrophy ${ }^{29}$ and adult-onset foveomacular vitelliform dystrophy. ${ }^{30} \mathrm{RPD}$ have been anecdotally reported in late-onset macular degeneration, ${ }^{31}$ vitamin $\mathrm{A}$ deficiency ${ }^{32}$ and IgA nephropathy. ${ }^{33}$

\section{Risk factors}

RPD occur in the setting of AMD, and, therefore, phenotypic features of AMD are associated with higher risk of RPD presence. Patients with late AMD have the highest odds of having RPD, and the risk is greater in the geographic atrophy (GA) over the CNV phenotype, followed by intermediate and early stages. ${ }^{4,5,34,35}$ Large drusen and focal pigmentary changes are additional risk factors for RPD presence. ${ }^{5}$ AMD and RPD share several non-ocular risk factors, including older age, ${ }^{5-7,36}$ female sex, ${ }^{5,7,19,36}$ current smoking ${ }^{6,7}$ and high body mass index. ${ }^{6}$ Other reported risk factors for RPD include less education, ${ }^{7}$ B-vitamin complex use, ${ }^{7}$ history of steroid eye drop use ${ }^{7}$ and glaucoma. ${ }^{7}$ As for AMD, the prevalence of RPD is lower in patients with diabetes. ${ }^{7,37}$ In the Beaver Dam Eye Study, ${ }^{7}$ RPD were an independent risk factor for mortality, and Cymerman et $\mathrm{al}^{38}$ have recently reported higher prevalence of RPD in patients with coronary artery disease. Compared with AMD without RPD, patients with RPD demonstrated higher rates of systemic hypertension, angina and mortality. ${ }^{39}$ However, this association is still under debate, and other studies did not confirm association between RPD and cardiovascular risk factors. ${ }^{40,41}$ Decreased renal function represents another vascular association; interestingly, both choroid and kidney share a lobular, end-arterial vascular system, and this could account for this connection. ${ }^{42}$

With the advent of the enhanced depth imaging (EDI)and swept-source (SS)-OCT, several studies focused on the choroidal imaging. A reduction of the subfoveal choroidal thickness has been extensively demonstrated in patients with RPD when compared not only with healthy subjects but also with AMD without RPD. ${ }^{43-46}$ Also, choroidal thinning involves all the macula area and even areas outside the macula, such as in the peripapillary region, suggesting a widespread choroidal involvement. ${ }^{47-50}$ Employing choroidal binarization, Corvi et $\mathrm{al}^{51}$ showed that stromal area is more represented in patients suffering from AMD with RPD compared with those without RPD, suggesting a choroidal 
vascular depletion and fibrotic replacement. Zheng et $\mathrm{al}^{52}$ illustrated choroidal vessel density reduction in eyes with RPD, suggesting that RPD may be a sign of choroidal vasculopathy. Interestingly, areas of lowest vessel density did not correspond to the location of thinnest choroid, and, therefore, RPD may be related to a global choroidal dysfunction leading to choriocapillaris hypoperfusion, possibly indicating an ocular sign of systemic vascular deficiency. ${ }^{52,53}$

Little is known regarding genetic susceptibility and RPD. Two major AMD risks alleles, namely, complement factor $\mathrm{H}(\mathrm{CHF}, \mathrm{rs} 1061170) 402 \mathrm{H}$ on chromosome 1q32 and the age-related maculopathy susceptibility 2 (ARMS2, rs10490924) 69S on chromosome 10q26, have been pointed out by large studies. ${ }^{54-57}$ Data regarding RPD are controversial. The Beaver Eye Study ${ }^{7}$ and the Blue Mountain Eye Study $^{6}$ revealed a linkage between RPD and both ARMS2 and $\mathrm{CFH}$ Y402H. Smith et $\mathrm{al}^{58}$ found ARMS2 and CHF $402 \mathrm{H}$ variant as a risk and protective factor, respectively. In the Melbourne Collaborative Cohort Study, ${ }^{5}$ the ARMS2 single-nucleotide polymorphism (SNP) rs10490924, HTRA1 SNPs rs11200638 and rs3793917, and CFH SNPs rs393955, rs1061170 and rs2274700 were linked to higher prevalence of RPD. Association between RPD and ARMS2 allele was also confirmed in Japanese population. ${ }^{17}$ Buitendijk et al ${ }^{9}$ found $\mathrm{CFH}, \mathrm{C} 2 / \mathrm{FB}$ and ARMS2 to be associated with both RPD and soft drusen, whereas C3 was linked only to RPD.

Conversely, Puche et $\mathrm{al}^{40}$ did not find any genotypic difference between AMD patients with and without RPD. Similarly, Boddu et $\mathrm{al}^{36}$ did not find any association between RPD and both ARMS2 and CHF alleles.

\section{Histology}

Different from drusen, which are made of lipids gathering in the BM (basal deposits), RPD are histologically characterized by the accumulation of material in the subretinal space extending up to the outer segment and even in the outer nuclear layers. ${ }^{59,60}$ Neighboring structures are affected by the presence of RPD, which is associated with RPE polymegathism, photoreceptor disruption and reactive gliosis. ${ }^{60}$

Although RPD have some similarities with drusen in their composition (ie, neutral lipid, cholesterol, amyloid, complement factor, and membranous debris), they have significant differences in their components, and, therefore, RPD are not just drusen material located above the RPE. ${ }^{60-63}$ RPD have higher concentration of unesterified cholesterol, vitronectin, include opsins, peanut agglutinin, and photoreceptor pigments (including precursors of A2E/lipofuscin) ${ }^{60-63}$ Since RPD and drusen lipids did not stain with the same dye, differences in lipid composition have been postulated. ${ }^{60}$ Moreover, RPD are rich in immune cells, predominantly immunereactive microglia and macrophages, confirming the role of inflammation. ${ }^{60}$ However, precise composition of RPD is yet to be determined.

\section{Imaging}

Different imaging modalities alone or in combination have been used to investigate RPD, including CFP, IR, short wavelength (SW)- and NIR-fundus autofluorescence (FAF), multicolor, OCT, en face OCT, OCT angiography (OCT-A), FA, indocyanine green angiography (ICGA) and adaptive optics. ${ }^{63}$ Figure 1 shows multimodal imaging of an eye with RPD. Written informed consent was received from all patients for publication of images in this review.

Multimodal imaging studies revealed that IR, FAF, SD-OCT and widefield en face SS-OCT have the highest sensitivity in detecting RPD, whereas late phases of ICGA, blue channel of CFP and confocal blue reflectance (CBR) have almost perfect specificity. ${ }^{14,18,64}$ However, there is no real gold standard for PRD detection, and use of at least 2 imaging modalities has been advocated. ${ }^{65}$ It has been suggested to use a high sensible modality (ie, SD-OCT, IR) as a screening test and a high specific one (ie, ICGA, CFP, CBR) as confirmation. ${ }^{18}$

\section{Color fundus photograph}

CFP reveals RPD as yellowish, ill-defined network of broad, interlacing ribbons preferentially located in the superior aspect of the macular area, which tends to become more punctate as they become closer to the fovea. ${ }^{59,66}$ The blue channel of CFP allows better contrast of RPD since the SW of blue light is highly reflected by the RPD, but preferentially absorbed by melanin contained in the RPE surrounding the RPD, creating contrast. ${ }^{18}$ Although some milestone studies ${ }^{5-7}$ employed CFP to individuate RPD, the diagnostic ability of this technique is unsatisfactory, due to the low sensitivity. ${ }^{18}$ On the contrary, its specificity is extremely high, virtually reaching $100 \%$, and, therefore, it could be a powerful confirmatory test. ${ }^{18}$

\section{Fundus autofluorescence}

SW-FAF (excitation $\lambda=488 \mathrm{~nm}$; emission $\lambda>500 \mathrm{~nm}$ ) reveals RPD as hypofluorescent. . $3,35,67-69$ The reason why RPD appear as hypofluorescent is still uncertain. ${ }^{18,70}$ One possible explanation is that the reflection and blockage of the blue excitation light prevent it from reaching the RPE.

On the other hand, most RPD deposits/subretinal materials may have an isofluorescent core conferring to these lesions a 

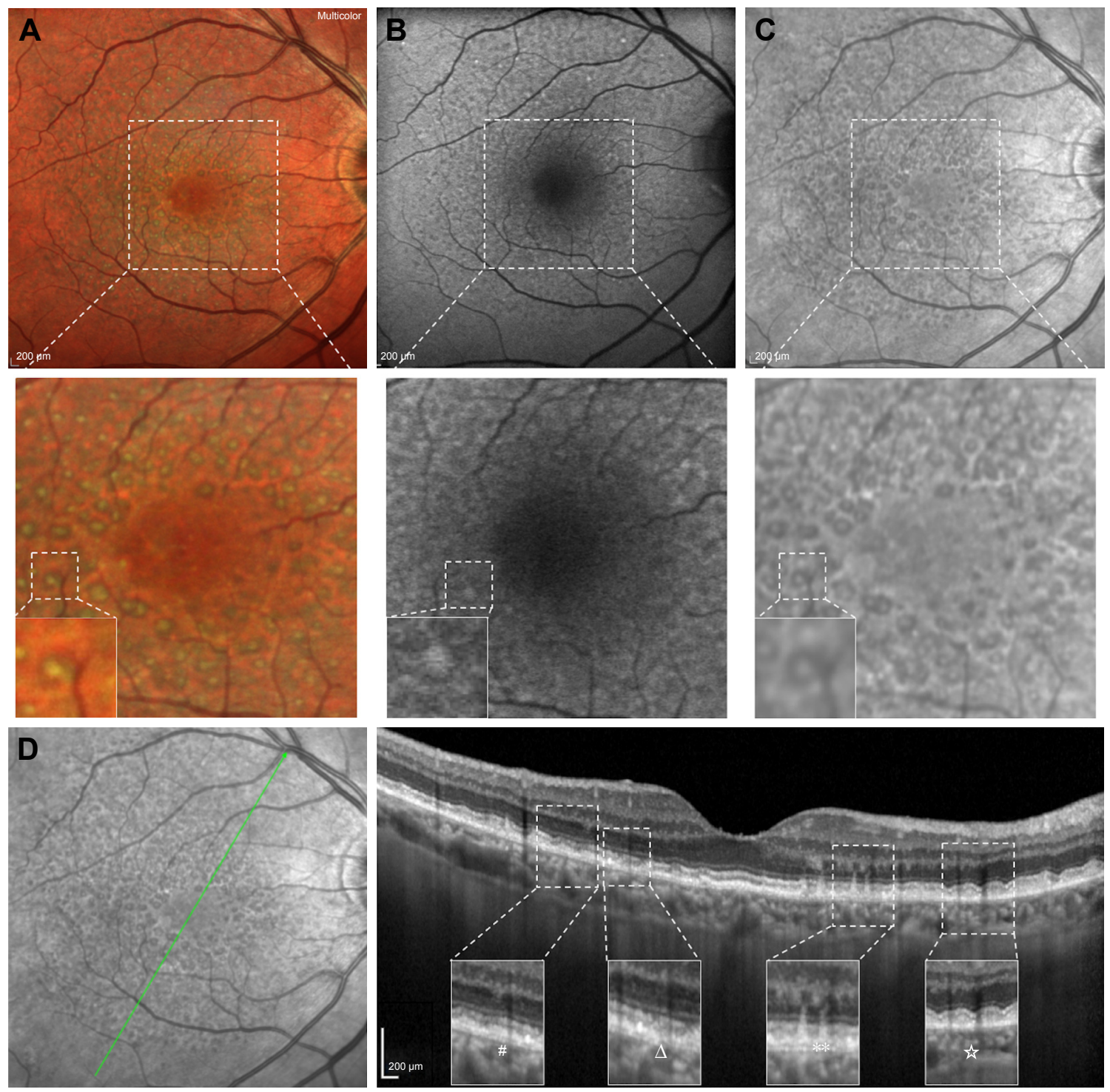

Figure I Multicolor imaging, FAF, IR and SD-OCT of a patient affected by RPD.

Notes: (A) Multicolor imaging showing a yellowish-green reticular pattern and some RPD with a target appearance characterized by a more intense yellowish/greenish core surrounded by a decreased intensity. (B) Blue FAF showing RPD as hypoautofluorescent dots surrounded by a faint hyperautofluorescent halo. Several RPD have a isoautofluorescent core, conferring a target aspect. (C) IR showing RPD as small lesions clustered in a reticular pattern and a variable target aspect, with an isoreflective core surrounded by hyporeflective halo. (D) Combined IR and structural SD-OCT B-scan passing through the fovea showing RPD as discrete accumulation of hyperreflective material above the RPE in the subretinal space. Green arrow shows the position of the scan lines used to generate the cross-sectional SD-OCT images. Magnifications showing an example of four different stages of RPD: I) diffuse accumulation of granular hyperreflective material between RPE and EZ (hash); 2) mounds of material bowing and distorting EZ profile (star); 3) conical accumulations with focal interruption of EZ (asterisk); 4) drusenoid material reabsorption and migration in inner retinal layers (triangle).

Abbreviations: FAF, fundus autofluorescence; IR, infrared reflectivity; SD-OCT, spectral-domain optical coherence tomography; RPD, reticular pseudodrusen; RPE, retinal pigment epithelium; EZ, ellipsoid zone.

"target aspect". ${ }^{24}$ This peculiar aspect could derive from the accumulation in the RPD core of lipofuscin-like material, probably representing unphagocytized photoreceptor outer segments; alternatively, it could be related to the interruption of ellipsoid zone (EZ). ${ }^{24}$ The absence of the target aspect in some RPD could be ascribed to heterogeneity in RPD composition or confocal selective laser ophthalmoscopy properties. ${ }^{24}$ Interestingly, a small subset of patients may have intense hyperfluorescent RPD, which have been initially reported as less associated with late AMD, ${ }^{71}$ even though it was not confirmed by later analysis. ${ }^{71,72}$ It has been speculated that hyperfluorescent RPD could be a transient form of pseudodrusen or small foci of acquired vitelliform material. ${ }^{71}$ RPD extent increases over time, and it has been estimated to have a mean growth rate of $4.4 \mathrm{~mm}^{2} /$ year. ${ }^{73}$ Using quantitative FAF (qFAF), it has been observed that eyes with RPD have lower qFAF values compared with patients with soft drusen, cuticular drusen and controls even in unaffected retina areas. ${ }^{74}$ 
Reduced qFAF values could reflect reduction in (intracellular but not extracellular) lipofuscin accumulation due to dysfunction in choroid-BM-RPE complex, slowing in visual cycle, reduced cone and rode densities and different composition of lipofuscin. ${ }^{74}$ Since it is rapid, noninvasive, highly reliable, accurate (sensitivity: 86\%, specificity: 92\%-95\%), SWFAF is a valuable imaging modality to identify RPD. ${ }^{18,68,75}$ Conversely, NIR-FAF (excitation $\lambda=787 \mathrm{~nm}$; emission $\lambda>800 \mathrm{~nm}$ ) has not good diagnostic abilities. ${ }^{18}$

\section{IR and multicolor}

RPD appearance at IR imaging parallels FAF showing a hyporeflective reticular pattern and a variable target aspect, with an isoreflective core surrounded by hyporeflective halo. . $4,35,73,76^{-10}$ Using multimodal imaging (including CFP, IR and OCT), Suzuki et al ${ }^{66}$ subclassified RPD in 3 different subtypes: dot, ribbon and midperipheral. In the first type, IR reveals perifoveal hyporeflective dots often showing a target aspect; the second type is characterized by perifoveal faint hyporeflective ribbons; and the third subtype is featured by midperipheral hyperreflective spots. ${ }^{66}$ Different RPD subtypes may have distinct components and may confer differing risk of disease progression. ${ }^{66}$ Since it is noninvasive, fast, high sensible and reliable, IR imaging has been advocated as a screening test to identify RPD. ${ }^{18}$

MultiColor (Spectralis SD-OCT; Heidelberg Engineering, Heidelberg, Germany) merges 3 different laser channels (ie, IR: $\lambda=820 \mathrm{~nm}$; green reflectance: $\lambda=515 \mathrm{~nm}$; blue reflectance at $\lambda=488 \mathrm{~nm}$ ) into a single pseudocolor image. RPD have a peculiar yellowish-green reticular pattern and are more evident at green and IR than at blue reflectance. ${ }^{77}$ Similar to IR, RPD may have a target appearance with a more intense yellowish/greenish core surrounded by a decreased intensity. ${ }^{75,77}$ MultiColor demonstrated equal identification rates of FAF and IR and higher individuation of target aspect. ${ }^{75,78}$ The green-blue enhanced mode, which stresses blue and green channels, reveals RPD as green/gray lesions, but has lower detection rates than standard MultiColor, confirming the value of the IR component. ${ }^{75}$

\section{OCT, en face OCT and OCT-A}

SD-OCT shows RPD as discrete accumulation of hyperreflective material above the RPE in the subretinal space. ${ }^{79,80}$ Although contradicted by some studies, it is widely accepted that that hyperreflective subretinal drusenoid material seen at SD-OCT corresponds to RPD seen at other imaging modalities. ${ }^{15,76,80,81}$ Zweifel et al ${ }^{80}$ cataloged RPD in 3 different stages: 1) diffuse accumulation of granular hyperreflective material between RPE and EZ, 2) mounds of material bowing and distorting EZ profile and 3) conical amassing with focal interruption of EZ. Subsequently, Querques et al $^{79}$ elucidated that RPD are dynamic structures characterized by agglomeration of subretinal material; moreover, they illustrated that the drusenoid material eventually reabsorbs and migrates into the inner retinal layers, and this was referred to as stage 4 . Auge et $\mathrm{al}^{82}$ confirmed the dynamism of RPD and emphasized the importance of dense scan protocols and exact registration of B-scans in the follow-up. Advanced stages of pseudodrusen life cycle are associated with sublesional RPE degeneration and shortening of photoreceptors. ${ }^{83}$ Small series reported that RPD can completely disappear during the time, and this phenomenon may be unilateral and asymmetric. ${ }^{84}$ RPD disappearance may leave outer retinal atrophy and focal reduction in choroidal thickness, and this could represent a novel phenotype of late AMD not included in the current classification systems. ${ }^{85}$ It has been speculated that outer retinal atrophy following RPD regression may eventually involve RPE and choriocapillaris, leading to GA. ${ }^{86}$

As previously discussed, several studies using EDI- and SS-OCT demonstrated that eyes with RPD are characterized by choroidal vascular depletion and fibrotic replacement, resulting in a widespread reduction in choroidal thickness even in comparison with other AMD phenotypes. ${ }^{43-52}$ Mrejen and Spaide ${ }^{87}$ observed that RPD have low prevalence in eyes with very thin choroid (ie, pathologic myopia), and, therefore, they argued that RPD accumulation is unlikely to be related to a primary dysfunction at the choroidal level, but rather explained by other factors (eg, RPE dysfunction). However, this argument fails to consider that the thin choroid in high myopia can be functionally and structurally healthy, compatible with normal visual function. Interestingly, high prevalence of RPD has been reported in eyes suffering from age-related choroidal atrophy, which is an entity recently described characterized by choroidal thickness reduction, pigmentary changes and scarcity of visible choroidal vessels, reflecting a disease of small vessels involving the choroid. ${ }^{88}$ Using OCT-A (Figure 2), eyes with RPD exhibited a reduction in choriocapillary vessel density more extensive than in those with drusen and no RPD and which has been associated with poor visual acuity. ${ }^{89-91}$

SD-OCT has excellent diagnostic properties and reliability in identification of RPD. ${ }^{18}$ Schaal et al ${ }^{64,92}$ demonstrated that en face OCT can image RPD, and this methodology alone has similar diagnostic abilities of conventional multimodal imaging (ie, CFP, IR and FAF). Polarization-sensitive (PS)-OCT is able to provide more contrast for structures, 

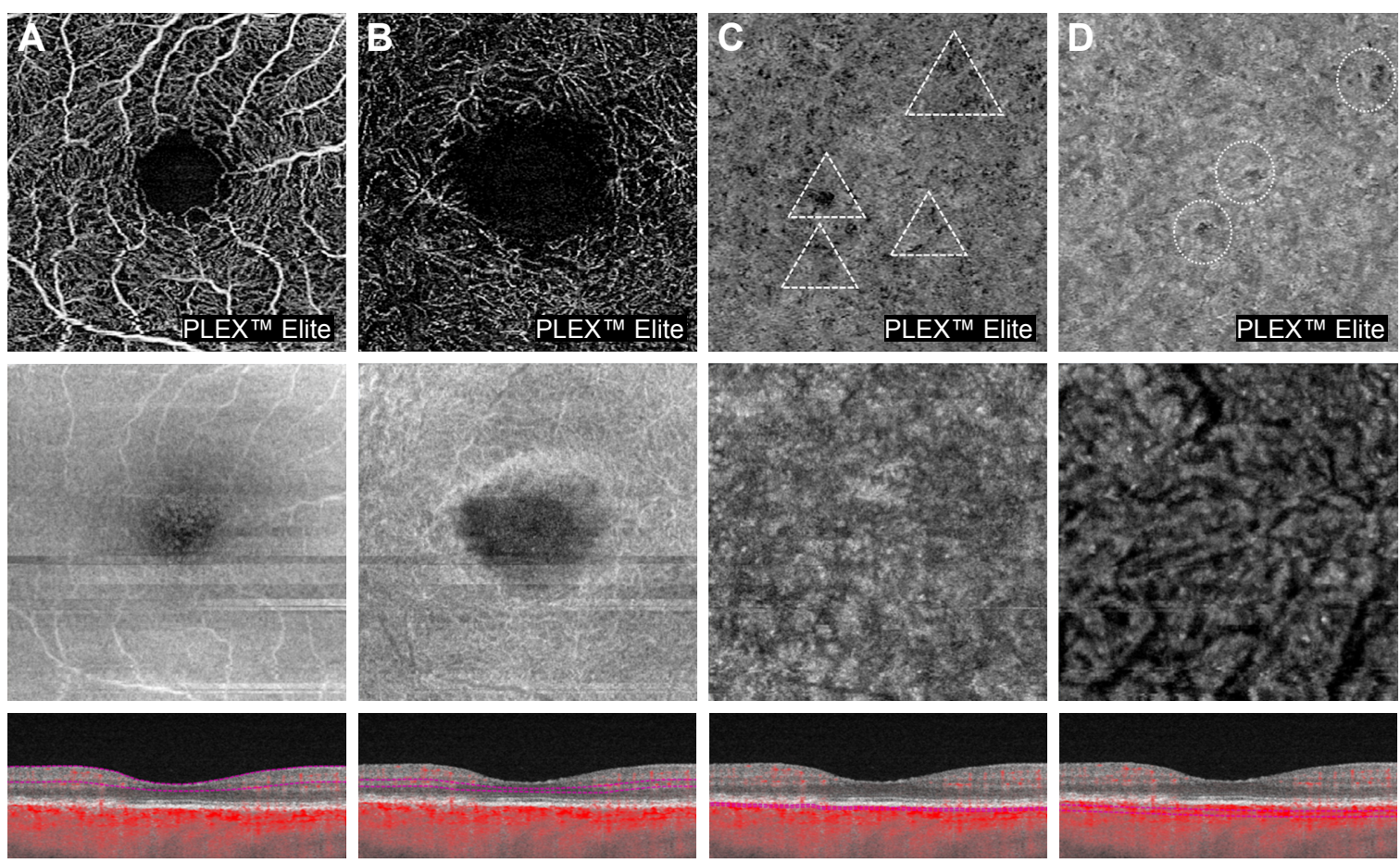

Figure 2 OCT-A of a patient affected by RPD.

Notes: (A, B) Superficial and deep capillary plexuses (A and B, respectively), corresponding en-face OCT and B-scan with flow showing no significant alterations. (C, D) Choriocapillaris and choroidal segmentation (C and $\mathbf{D}$, respectively) showing choriocapillaris and choroidal impairment of the vessel density (dashed triangles and circles, respectively). Measurement: $3 \times 3 \mathrm{~mm}$ OCT-A.

Abbreviations: OCT-A, optical coherence tomography angiography; RPD, reticular pseudodrusen; OCT, optical coherence tomography.

such as RPE, that are able to alter the polarization state of the light. ${ }^{93}$ PS-OCT can identify RPD, which preserve polarization, and distinguish them from drusen, which, conversely, alter polarization. ${ }^{94}$ However, PS-OCT has not broken into the clinical practice, and its diagnostic properties are still unknown.

In addition to RPD identification, some authors have tried to determine whether OCT could provide information on the natural history of the disease. RPD thickness was a robust biomarker of future areas of GA growth. ${ }^{95-97}$ Moreover, EZ disruption pattern has been related to GA progression over time. ${ }^{98}$ Identification of biomarkers and anatomic surrogates predicting future vision loss could be beneficial for clinical trials concerning early or intermediate AMD. ${ }^{99}$

\section{FA and ICGA}

FA may reveal RPD as a filling defect in the choriocapillaris in early frames; however, due to its low sensitivity and invasive nature, it is seldom performed. ${ }^{63}$

RPD are seen as hypofluorescent dots at mid and late phases of ICGA, which co-localize with dots seen at IR and FAF, suggesting a pathology internal to RPE. ${ }^{14,100}$ Using IR and ICGA, Querques et $\mathrm{al}^{44}$ showed that RPD co-localize with choroidal intervascular stroma with sparing of areas above large choroidal vessels, suggesting impaired choroidal filling. Similarly, Alten et a ${ }^{101}$ observed that RPD are located in areas of choroidal watershed, reinforcing the pathogenic role of choroidal hypoxia. However, further study did not find concordance between RPD and large choroidal blood vessels. ${ }^{102}$ Although it owns good diagnostic abilities (sensitivity: $73 \%$, specificity: $100 \%$ ), ICGA is not the first choice test because of its invasive nature. ${ }^{18}$ As shown in Figure 3, FA and ICGA have a pivotal role in diagnosing CNV associated with RPD.

\section{Adaptive optics}

By improving the transverse resolution to $2 \mu \mathrm{m}$, adaptive optics visualize cone photoreceptors in vivo. Adaptive optics illustrate RPD as isoreflective lesions surrounded by a continuous/discontinuous hyporeflective halo, with the hyperreflective core ascribed to the drusenoid material itself and the hyporeflective halo related to absent or degenerated photoreceptors correspondent to EZ disruption. ${ }^{103}$ Photoreceptors mosaic is perturbed by RPD according to lesion stage; specifically, stages 1 and 2 are characterized by a reduction in photoreceptor density, whereas only stage 3 has an annular 

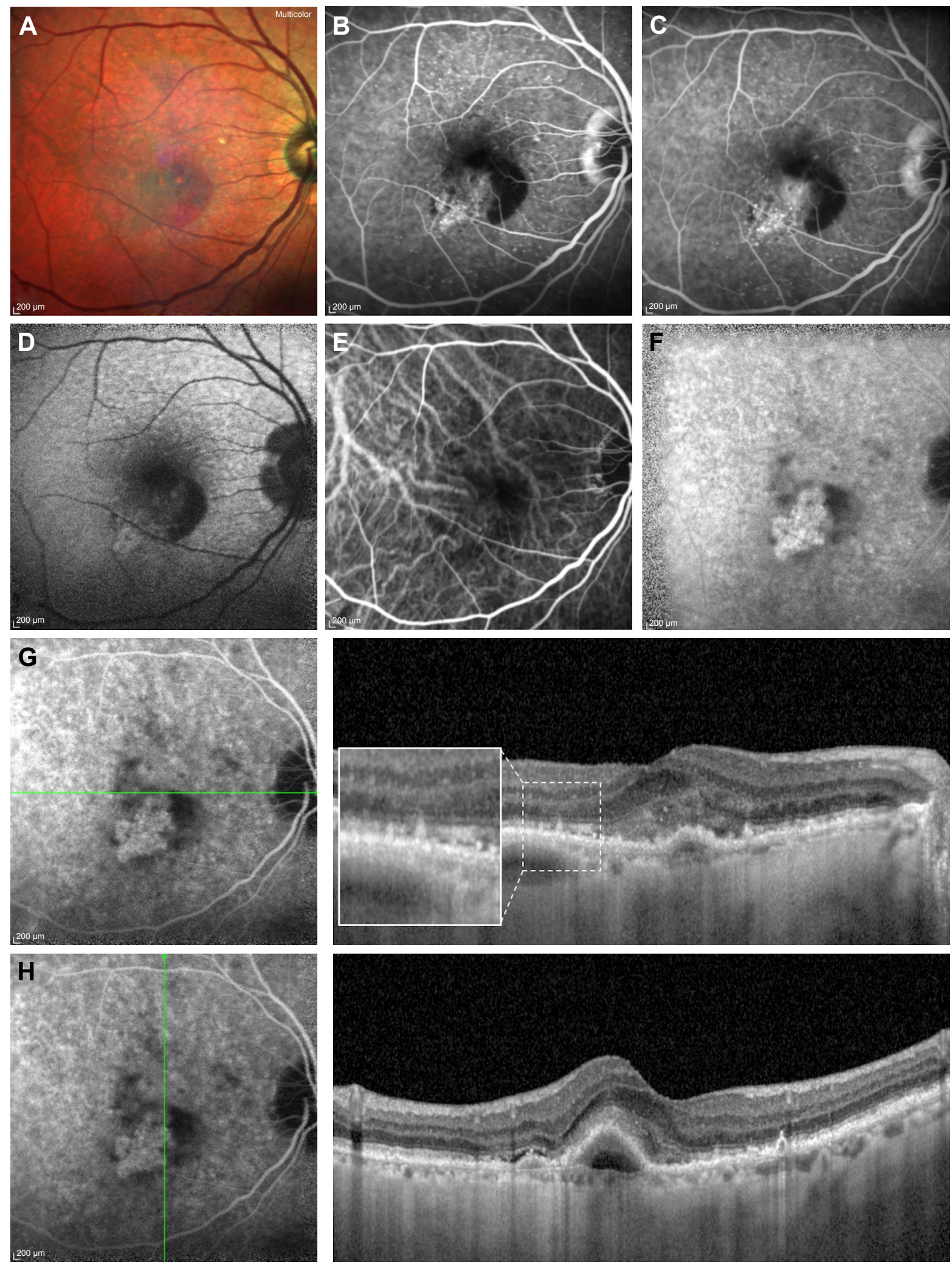

Figure 3 Multicolor imaging, FA, fundus autofluorescence, ICGA and structural SD-OCT of a patient affected by RPD and a type I CNV.

Notes: (A, D) Multicolor imaging (A) and fundus autofluorescence (D) showing RPD, a large hemorrhage around the central lesion. FA (B, C) and ICGA (E, F) revealing the presence of a type I CNV with a typical hyperfluorescent plaque in the late phase of ICGA (F). (G, H) Combined ICGA and structural horizontal and vertical SD-OCT B-scans passing through the fovea revealing the presence of RPD and an active type I CNV. Green arrows show the position of the scan lines used to generate the crosssectional SD-OCT images.

Abbreviations: FA, fluorescein angiography; ICGA, indocyanine green angiography; SD-OCT, spectral-domain optical coherence tomography; RPD, reticular pseudodrusen; $\mathrm{CNV}$, choroidal neovascularization. 
configuration. ${ }^{104,105}$ As shown by OCT studies, dynamism of RPD was further confirmed using adaptive optics. ${ }^{106}$

Mrejen et $\mathrm{al}^{107}$ showed that cone density over and around RPD is lower than drusen and healthy controls, suggesting that RPD could affect retinal function even in the absence of CNV or GA. On the contrary, Querques et $\mathrm{al}^{103}$ found that cone density similarly decreased in drusen and RPD.

\section{Functional tests}

AMD is characterized by abnormal retinal function even in early or intermediate stage. Impairment in dark adaption and low-luminance visual acuity have been demonstrated in AMD, and these parameters are more severely affected by the presence of RPD potentially reflecting impairment in rod function. ${ }^{68,108,109}$ In agreement with this hypothesis, Steinberg et a ${ }^{110}$ illustrated a more pronounced deterioration of mean threshold sensitivity at scotopic than at photopic microperimetry. A recent study comparing eyes in normal macular health with and without RPD illustrated no significant difference regarding photopic acuity, mesopic acuity, low-luminance acuity, photopic contrast sensitivity and photopic light sensitivity. ${ }^{111}$ In the same study, eyes with RPD exhibited delayed dark adaptation, although significant differences in age between the 2 groups could be imputable for such a difference. ${ }^{111}$

Eyes with RPD have reduction in microperimetric retinal sensitivity despite preserved visual acuity. ${ }^{112}$ Compared with drusen, RPD are associated with a more pronounced impairment in macular sensitivity. ${ }^{113}$ Whether the extent of RPD is related to macular sensitivity is still unclear and contrasting results have been published. ${ }^{114,115}$

By recording local electroretinographic responses from the central $40^{\circ}-50^{\circ}$ of the macula, multifocal electroretinography (mfERG) allows assessing retinal function in a topographic way. Studies involving mfERG reported contrasting results. ${ }^{115-117}$ Alten et a ${ }^{116,117}$ did not find any significant difference between patients with RPD and healthy controls. Comparing areas with and without pseudodrusen, they observed no significant differences at baseline; conversely, reduction in amplitude was seen in affected areas during the follow-up, suggesting a progressive decline in retinal function over time, which did not correlate with structural data. ${ }^{116,117} \mathrm{Wu}$ et al ${ }^{115}$ reported conflicting results, showing that RPD presence and extent in intermediate AMD are independently linked to worse mfERG implicit time, but not to amplitude.

\section{Natural history}

As illustrated in the aforementioned paragraphs, RPD are dynamic structures evolving and even reabsorbing with time. RPD confer a higher risk of developing AMD during follow-up. In elder population with an otherwise healthy macula, RPD were found in the $25 \%$ of subjects conferring a 2-fold risk to develop early AMD. ${ }^{118}$ In addition, the presence of RPD increase is significantly associated with higher odds to progress to late AMD. ${ }^{6,119}$ In fellow eyes of patients with unilateral CNV, the presence of AMD conferred extra odds to progress to late $\mathrm{AMD} .^{34} \mathrm{RPD}$ are linked to the development of GA, especially with multilobular type, and higher progression rates. ${ }^{96,97,120} \mathrm{RPD}$ are a strong risk factor for developing type $3 \mathrm{CNV}$, and, moreover, they are associated with higher earlier onset and bilateral disease. ${ }^{121,122}$ Eyes affected by type $3 \mathrm{CNV}$ have higher odds to have also RPD, and, for this reason, RPD have been advocated as a diagnostic sign to distinguish type $3 \mathrm{CNV}$ from other forms. ${ }^{123,124}$ Eyes with exclusively RPD have been associated with type $2 \mathrm{CNV} .{ }^{125}$ In case of CNV development, RPD fade nearby the CNV itself, but they may still be observed more peripherally. ${ }^{14,69}$

RPD are a risk factor to develop atrophy following intravitreal anti-vascular endothelial growth factor (VEGF). ${ }^{126-128}$ However, presence of RPD did not interfere with the response to intravitreal anti-VEGF, at least in the short term. ${ }^{129}$

\section{Therapy}

No specific therapy is available for RPD. Since RPD are in the spectrum of AMD, they can benefit from vitamin supplementation (ie, AREDS and AREDS2 formula) in case of early and intermediate diseases. ${ }^{130}$ Beyond reducing the risk of late AMD development, supplementation with macular pigment component (ie, lutein, zeaxanthin and meso-zeaxanthin) may increase the macular pigment optical density primarily, if not only, in the RPD phenotype. ${ }^{131}$ Presence of RPD in the setting of late AMD has no impact on the therapeutic strategy.

\section{Conclusions}

RPD represent a specific component of AMD, although they may be found even in other ocular diseases. They differ from drusen not only for their location (ie, subretinal space) but also for their components. Their presence should always be sought in patients suffering from AMD, since they are strongly associated with late stages of disease. RPD identification should employ at least 2 different imaging modalities, since their recognition may not be straightforward with one technique only. Future clinical trials and epidemiological studies should take into account RPD phenotype employing imaging modalities suitable for their identification.

\section{Disclosure}

GQ is a consultant for Alimera Sciences (Alpharetta, Georgia, USA), Allergan Inc. (Irvine, California, USA), Bayer 
Schering-Pharma (Berlin, Germany), Heidelberg (Germany), Novartis (Basel, Switzerland), Sandoz (Berlin, Germany), Zeiss (Dublin, OH, USA). FB has the following disclosures: Allergan (s), Alimera (s), Bayer (s), Farmila-Thea (s), Schering Pharma (s), Sanofi-Aventis (s), Novagali (s), Pharma (s), Hoffmann-La Roche (s), Genentech (s) and Novartis (s). The other authors report no conflicts of interest in this work.

\section{References}

1. Lim LS, Mitchell P, Seddon JM, Holz FG, Wong TY. Age-related macular degeneration. Lancet. 2012;379(9827):1728-1738.

2. Mimoun G, Soubrane G, Coscas G. Les drusen maculaires. [Macular drusen]. J Fr Ophtalmol. 1990;13(10):511-530. French.

3. Klein R, Davis MD, Magli YL, Segal P, Klein BE, Hubbard L. The Wisconsin age-related maculopathy grading system. Ophthalmology. 1991;98(7):1128-1134.

4. Arnold JJ, Sarks SH, Killingsworth MC, Sarks JP. Reticular pseudodrusen. A risk factor in age-related maculopathy. Retina. 1995;15(3): 183-191.

5. Finger RP, Chong E, McGuinness MB, et al. Reticular Pseudodrusen and Their Association with Age-Related Macular Degeneration: The Melbourne Collaborative Cohort Study. Ophthalmology. 2016; 123(3):599-608.

6. Joachim N, Mitchell P, Rochtchina E, Tan AG, Wang JJ. Incidence and progression of reticular drusen in age-related macular degeneration: findings from an older Australian cohort. Ophthalmology. 2014; 121(4):917-925.

7. Klein R, Meuer SM, Knudtson MD, Iyengar SK, Klein BE. The epidemiology of retinal reticular drusen. Am J Ophthalmol. 2008;145(2): 317-326.

8. De Bats F, Mathis T, Mauget-Faysse M, Joubert F, Denis P, Kodjikian L. Prevalence of reticular pseudodrusen in age-related macular degeneration using multimodal imaging. Retina. 2016;36(1):46-52.

9. Buitendijk GH, Hooghart AJ, Brussee C, et al. Epidemiology of reticular pseudodrusen in age-related macular degeneration: the Rotterdam study. Invest Ophthalmol Vis Sci. 2016;57(13):5593-5601.

10. Chan H, Cougnard-Gregoire A, Delyfer MN, et al. Multimodal imaging of reticular pseudodrusen in a population-based setting: the alienor study. Invest Ophthalmol Vis Sci. 2016;57(7):3058-3065.

11. Zarubina AV, Neely DC, Clark ME, et al. Prevalence of subretinal drusenoid deposits in older persons with and without age-related macular degeneration, by multimodal imaging. Ophthalmology. 2016;123(5): 1090-1100.

12. Joachim N, Mitchell P, Younan C, et al. Ethnic variation in early agerelated macular degeneration lesions between white Australians and Singaporean Asians. Invest Ophthalmol Vis Sci. 2014;55(7):4421-4429.

13. Alten F, Clemens CR, Milojcic C, Eter N. Subretinal drusenoid deposits associated with pigment epithelium detachment in age-related macular degeneration. Retina. 2012;32(9):1727-1732.

14. Smith RT, Sohrab MA, Busuioc M, Barile G. Reticular macular disease. Am J Ophthalmol. 2009;148(5):733-743.e2.

15. Sohrab MA, Smith RT, Salehi-Had H, Sadda SR, Fawzi AA. Image registration and multimodal imaging of reticular pseudodrusen. Invest Ophthalmol Vis Sci. 2011;52(8):5743-5748.

16. Cohen SY, Dubois L, Tadayoni R, Delahaye-Mazza C, Debibie C, Quentel G. Prevalence of reticular pseudodrusen in age-related macular degeneration with newly diagnosed choroidal neovascularisation. Br J Ophthalmol. 2007;91(3):354-359.

17. Ueda-Arakawa N, Ooto S, Nakata I, et al. Prevalence and genomic association of reticular pseudodrusen in age-related macular degeneration. Am J Ophthalmol. 2013;155(2):260-269.e262.

18. Ueda-Arakawa N, Ooto S, Tsujikawa A, Yamashiro K, Oishi A, Yoshimura N. Sensitivity and specificity of detecting reticular pseudodrusen in multimodal imaging in Japanese patients. Retina. 2013;33(3): 490-497.
19. Wilde C, Patel M, Lakshmanan A, Morales MA, Dhar-Munshi S, Amoaku WM. Prevalence of reticular pseudodrusen in eyes with newly presenting neovascular age-related macular degeneration. Eur J Ophthalmol. 2016;26(2):128-134.

20. Wu Z, Ayton LN, Luu CD, Baird PN, Guymer RH. Reticular pseudodrusen in intermediate age-related macular degeneration: prevalence, detection, clinical, environmental, and genetic associations. Invest Ophthalmol Vis Sci. 2016;57(3):1310-1316.

21. Curcio CA, Messinger JD, Sloan KR, McGwin G, Medeiros NE, Spaide RF. Subretinal drusenoid deposits in non-neovascular agerelated macular degeneration: morphology, prevalence, topography, and biogenesis model. Retina. 2013;33(2):265-276.

22. Kim JH, Chang YS, Kim JW, Lee TG, Kim CG. Prevalence of subtypes of reticular pseudodrusen in newly diagnosed exudative age-related macular degeneration and polypoidal choroidal vasculopathy in Korean patients. Retina. 2015;35(12):2604-2612.

23. Smith RT, Chan JK, Busuoic M, Sivagnanavel V, Bird AC, Chong NV. Autofluorescence characteristics of early, atrophic, and high-risk fellow eyes in age-related macular degeneration. Invest Ophthalmol Vis Sci. 2006;47(12):5495-5504.

24. Querques G, Querques L, Martinelli D, et al. Pathologic insights from integrated imaging of reticular pseudodrusen in age-related macular degeneration. Retina. 2011;31(3):518-526.

25. Zweifel SA, Spaide RF, Yannuzzi LA. Acquired vitelliform detachment in patients with subretinal drusenoid deposits (reticular pseudodrusen). Retina. 2011;31(2):229-234.

26. De Bats F, Wolff B, Mauget-Faysse M, Meunier I, Denis P, Kodjikian L. Association of reticular pseudodrusen and early onset drusen. ISRN Ophthalmol. 2013;2013:273085.

27. Gliem M, Muller PL, Birtel J, Hendig D, Holz FG, Charbel Issa P. Frequency, phenotypic characteristics and progression of atrophy associated with a diseased bruch's membrane in pseudoxanthoma elasticum. Invest Ophthalmol Vis Sci. 2016;57(7):3323-3330.

28. Zweifel SA, Imamura Y, Freund KB, Spaide RF. Multimodal fundus imaging of pseudoxanthoma elasticum. Retina. 2011;31(3):482-491.

29. Gliem M, Muller PL, Mangold E, et al. Reticular pseudodrusen in sorsby fundus dystrophy. Ophthalmology. 2015;122(8):1555-1562.

30. Wilde C, Lakshmanan A, Patel M, Morales MU, Dhar-Munshi S, Amoaku WM. Prevalence of reticular pseudodrusen in newly presenting adult onset foveomacular vitelliform dystrophy. Eye (Lond). 2016;30(6):817-824.

31. Cukras C, Flamendorf J, Wong WT, Ayyagari R, Cunningham D, Sieving PA. Longitudinal structural changes in late-onset retinal degeneration. Retina. 2016;36(12):2348-2356.

32. Aleman TS, Garrity ST, Brucker AJ. Retinal structure in vitamin A deficiency as explored with multimodal imaging. Doc Ophthalmol. 2013;127(3):239-243.

33. Lally DR, Baumal C. Subretinal drusenoid deposits associated with complement-mediated IgA nephropathy. JAMA Ophthalmol. 2014; 132(6):775-777.

34. Pumariega NM, Smith RT, Sohrab MA, Letien V, Souied EH. A prospective study of reticular macular disease. Ophthalmology. 2011;118(8): 1619-1625.

35. Schmitz-Valckenberg S, Alten F, Steinberg JS, et al; Geographic Atrophy Progression (GAP) Study Group. Reticular drusen associated with geographic atrophy in age-related macular degeneration. Invest Ophthalmol Vis Sci. 2011;52(9):5009-5015.

36. Boddu S, Lee MD, Marsiglia M, Marmor M, Freund KB, Smith RT. Risk factors associated with reticular pseudodrusen versus large soft drusen. Am J Ophthalmol. 2014;157(5):985-993.e2.

37. Cho BJ, Heo JW, Shin JP, Ahn J, Kim TW, Chung H. Epidemiological association between systemic diseases and age-related macular degeneration: the Korea National Health and Nutrition Examination Survey 2008-2011. Invest Ophthalmol Vis Sci. 2014;55(7):4430-4437.

38. Cymerman RM, Skolnick AH, Cole WJ, Nabati C, Curcio CA, Smith RT. Coronary artery disease and reticular macular disease, a subphenotype of early age-related macular degeneration. Curr Eye Res. 2016;41(11): $1482-1488$. 
39. Rastogi N, Smith RT. Association of age-related macular degeneration and reticular macular disease with cardiovascular disease. Surv Ophthalmol. 2016;61(4):422-433.

40. Puche N, Blanco-Garavito R, Richard F, et al. Genetic and environmental factors associated with reticular pseudodrusen in age-related macular degeneration. Retina. 2013;33(5):998-1004.

41. McCarter RV, McKay GJ, Quinn NB, et al. Evaluation of coronary artery disease as a risk factor for reticular pseudodrusen. Br J Ophthalmol. Epub 2017 Aug 19.

42. Leisy HB, Ahmad M, Marmor M, Smith RT. Association between decreased renal function and reticular macular disease in age-related macular degeneration. Ophthalmology Retina. 2017;1(1):42-48.

43. Garg A, Oll M, Yzer S, et al. Reticular pseudodrusen in early age-related macular degeneration are associated with choroidal thinning. Invest Ophthalmol Vis Sci. 2013;54(10):7075-7081.

44. Querques G, Querques L, Forte R, Massamba N, Coscas F, Souied EH. Choroidal changes associated with reticular pseudodrusen. Invest Ophthalmol Vis Sci. 2012;53(3):1258-1263.

45. Thorell MR, Goldhardt R, Nunes RP, et al. Association between subfoveal choroidal thickness, reticular pseudodrusen, and geographic atrophy in age-related macular degeneration. Ophthalmic Surg Lasers Imaging Retina. 2015;46(5):513-521.

46. Switzer DW Jr, Mendonca LS, Saito M, Zweifel SA, Spaide RF. Segregation of ophthalmoscopic characteristics according to choroidal thickness in patients with early age-related macular degeneration. Retina. 2012;32(7):1265-1271.

47. Yun C, Oh J, Ahn SE, Hwang SY, Kim SW, Huh K. Peripapillary choroidal thickness in patients with early age-related macular degeneration and reticular pseudodrusen. Graefes Arch Clin Exp Ophthalmol. 2016;254(3):427-435.

48. Haas P, Esmaeelpour M, Ansari-Shahrezaei S, Drexler W, Binder S. Choroidal thickness in patients with reticular pseudodrusen using 3D 1060-nm OCT maps. Invest Ophthalmol Vis Sci. 2014;55(4): 2674-2681.

49. Ueda-Arakawa N, Ooto S, Ellabban AA, et al. Macular choroidal thickness and volume of eyes with reticular pseudodrusen using sweptsource optical coherence tomography. Am J Ophthalmol. 2014;157(5): 994-1004.

50. Capuano V, Souied EH, Miere A, Jung C, Costanzo E, Querques G. Choroidal maps in non-exudative age-related macular degeneration. Br J Ophthalmol. 2016;100(5):677-682.

51. Corvi F, Souied EH, Capuano V, et al. Choroidal structure in eyes with drusen and reticular pseudodrusen determined by binarisation of optical coherence tomographic images. Br J Ophthalmol. 2017;101:348-352.

52. Zheng F, Gregori G, Schaal KB, et al. Choroidal thickness and choroidal vessel density in nonexudative age-related macular degeneration using swept-source optical coherence tomography imaging. Invest Ophthalmol Vis Sci. 2016;57(14):6256-6264.

53. A Martillo M, Marsiglia M, D Lee M, Pumariega N, Bearelly S, Smith RT. Is reticular macular disease a choriocapillaris perfusion problem? Med Hypothesis Discov Innov Ophthalmol. 2012;1(2):37-41.

54. Edwards AO, Ritter R 3rd, Abel KJ, Manning A, Panhuysen C, Farrer LA. Complement factor $\mathrm{H}$ polymorphism and age-related macular degeneration. Science. 2005;308(5720):421-424.

55. Jakobsdottir J, Conley YP, Weeks DE, Mah TS, Ferrell RE, Gorin MB. Susceptibility genes for age-related maculopathy on chromosome 10q26. Am J Hum Genet. 2005;77(3):389-407.

56. Klein RJ, Zeiss C, Chew EY, et al. Complement factor H polymorphism in age-related macular degeneration. Science. 2005;308(5720): 385-389.

57. Rivera A, Fisher SA, Fritsche LG, et al. Hypothetical LOC387715 is a second major susceptibility gene for age-related macular degeneration, contributing independently of complement factor $\mathrm{H}$ to disease risk. Hum Mol Genet. 2005;14(21):3227-3236.

58. Smith RT, Merriam JE, Sohrab MA, et al. Complement factor $\mathrm{H} 402 \mathrm{H}$ variant and reticular macular disease. Arch Ophthalmol. 2011;129(8): 1061-1066.
59. Khan KN, Mahroo OA, Khan RS, et al. Differentiating drusen: Drusen and drusen-like appearances associated with ageing, age-related macular degeneration, inherited eye disease and other pathological processes. Prog Retin Eye Res. 2016;53:70-106.

60. Greferath U, Guymer RH, Vessey KA, Brassington K, Fletcher EL. Correlation of histologic features with in vivo imaging of reticular pseudodrusen. Ophthalmology. 2016;123(6):1320-1331.

61. Curcio CA, Presley JB, Millican CL, Medeiros NE. Basal deposits and drusen in eyes with age-related maculopathy: evidence for solid lipid particles. Exp Eye Res. 2005;80(6):761-775.

62. Johnson LV, Leitner WP, Staples MK, Anderson DH. Complement activation and inflammatory processes in Drusen formation and age related macular degeneration. Exp Eye Res. 2001;73(6):887-896.

63. Sivaprasad S, Bird A, Nitiahpapand R, et al. Perspectives on reticular pseudodrusen in age-related macular degeneration. Surv Ophthalmol. 2016;61(5):521-537.

64. Schaal KB, Legarreta AD, Feuer WJ, et al. Comparison between widefield en face swept-source OCT and conventional multimodal imaging for the detection of reticular pseudodrusen. Ophthalmology. 2017; 124(2):205-214.

65. Alten F, Eter N. Current knowledge on reticular pseudodrusen in agerelated macular degeneration. Br J Ophthalmol. 2015;99(6):717-722.

66. Suzuki M, Sato T, Spaide RF. Pseudodrusen subtypes as delineated by multimodal imaging of the fundus. Am J Ophthalmol. 2014;157(5): 1005-1012.

67. Bindewald A, Bird AC, Dandekar SS, et al. Classification of fundus autofluorescence patterns in early age-related macular disease. Invest Ophthalmol Vis Sci. 2005;46(9):3309-3314.

68. Hogg RE, Silva R, Staurenghi G, et al. Clinical characteristics of reticular pseudodrusen in the fellow eye of patients with unilateral neovascular age-related macular degeneration. Ophthalmology. 2014;121(9): 1748-1755.

69. Sarks J, Arnold J, Ho IV, Sarks S, Killingsworth M. Evolution of reticular pseudodrusen. Br J Ophthalmol. 2011;95(7):979-985.

70. Lois N, Owens SL, Coco R, Hopkins J, Fitzke FW, Bird AC. Fundus autofluorescence in patients with age-related macular degeneration and high risk of visual loss. Am J Ophthalmol. 2002;133(3):341-349.

71. Lee MY, Ham DI. Subretinal drusenoid deposits with increased autofluorescence in eyes with reticular pseudodrusen. Retina. 2014; 34(1):69-76.

72. Querques G, Souied EH. Correspondence. Retina. 2015;35(1):e4-e6.

73. Steinberg JS, Auge J, Jaffe GJ, Fleckenstein M, Holz FG, SchmitzValckenberg S; GAP Study Group. Longitudinal analysis of reticular drusen associated with geographic atrophy in age-related macular degeneration. Invest Ophthalmol Vis Sci. 2013;54(6):4054-4060.

74. Gliem M, Muller PL, Finger RP, McGuinness MB, Holz FG, Charbel Issa P. Quantitative fundus autofluorescence in early and intermediate age-related macular degeneration. JAMA Ophthalmol. 2016; 134(7):817-824.

75. Alten F, Clemens CR, Heiduschka P, Eter N. Characterisation of reticular pseudodrusen and their central target aspect in multi-spectral, confocal scanning laser ophthalmoscopy. Graefes Arch Clin Exp Ophthalmol. 2014;252(5):715-721.

76. Spaide RF. Colocalization of pseudodrusen and subretinal drusenoid deposits using high-density en face spectral domain optical coherence tomography. Retina. 2014;34(12):2336-2345.

77. Querques G, Srour M, Massamba N, Puche N, Souied EH. Reticular pseudodrusen. Ophthalmology. 2013;120(4):872.

78. Badal J, Biarnes M, Mones J. Performance characteristics of multicolor versus blue light and infrared imaging in the identification of reticular pseudodrusen. Int Ophthalmol. Epub 2017 Jan 21.

79. Querques G, Canoui-Poitrine F, Coscas F, et al. Analysis of progression of reticular pseudodrusen by spectral domain-optical coherence tomography. Invest Ophthalmol Vis Sci. 2012;53(3):1264-1270.

80. Zweifel SA, Spaide RF, Curcio CA, Malek G, Imamura Y. Reticular pseudodrusen are subretinal drusenoid deposits. Ophthalmology. 2010; 117(2):303-312. e1. 
81. Heiferman MJ, Fernandes JK, Munk M, Mirza RG, Jampol LM, Fawzi AA. Reticular pseudodrusen on infrared imaging are topographically distinct from subretinal drusenoid deposits on en face optical coherence tomography. Retina. 2015;35(12):2593-2603.

82. Auge J, Steinberg JS, Fleckenstein M, Holz FG, Schmitz-Valckenberg S. Retikuläre Drusen über die Zeit mittels SD-OCT. [Reticular drusen over time with SD-OCT]. Ophthalmologe. 2014;111(8):765-771. German.

83. Xu X, Liu X, Wang X, et al. Retinal pigment epithelium degeneration associated with subretinal drusenoid deposits in age-related macular degeneration. Am J Ophthalmol. 2017;175:87-98.

84. Schick T, Ersoy L, Kirchhof B, Liakopoulos S. Asymmetrical behaviour of disappearance of reticular pseudodrusen in both eyes during long-term follow-up with spectral domain optical coherence tomography. GMS Ophthalmol Cases. 2014;4:Doc06.

85. Spaide RF. Outer retinal atrophy after regression of subretinal drusenoid deposits as a newly recognized form of late age-related macular degeneration. Retina. 2013;33(9):1800-1808.

86. Spaide RF. Improving the age-related macular degeneration construct: a new classification system. Retina. Epub 2017 May 26.

87. Mrejen S, Spaide RF. The relationship between pseudodrusen and choroidal thickness. Retina. 2014;34(8):1560-1566.

88. Spaide RF. Age-related choroidal atrophy. Am J Ophthalmol. 2009; 147(5):801-810.

89. Alten F, Heiduschka P, Clemens CR, Eter N. Exploring choriocapillaris under reticular pseudodrusen using OCT-angiography. Graefes Arch Clin Exp Ophthalmol. 2016;254(11):2165-2173.

90. Cicinelli MV, Rabiolo A, Marchese A, et al. Choroid morphometric analysis in non-neovascular age-related macular degeneration by means of optical coherence tomography angiography. Br J Ophthalmol 2017;101(9):1193-1200.

91. Nesper PL, Soetikno BT, Fawzi AA. Choriocapillaris nonperfusion is associated with poor visual acuity in eyes with reticular pseudodrusen. Am J Ophthalmol. 2017;174:42-55.

92. Schaal KB, Legarreta AD, Gregori G, et al. Widefield en face optical coherence tomography imaging of subretinal drusenoid deposits. Ophthalmic Surg Lasers Imaging Retina. 2015;46(5):550-559.

93. Pircher M, Hitzenberger CK, Schmidt-Erfurth U. Polarization sensitive optical coherence tomography in the human eye. Prog Retin Eye Res. 2011;30(6):431-451.

94. Roberts PK, Baumann B, Schlanitz FG, et al. Retinal pigment epithelial features indicative of neovascular progression in age-related macular degeneration. Br J Ophthalmol. Epub 2017 Mar 7.

95. Niu S, de Sisternes L, Chen Q, Rubin DL, Leng T. Fully automated prediction of geographic atrophy growth using quantitative spectraldomain optical coherence tomography biomarkers. Ophthalmology. 2016;123(8):1737-1750.

96. Marsiglia M, Boddu S, Bearelly S, et al. Association between geographic atrophy progression and reticular pseudodrusen in eyes with dry age-related macular degeneration. Invest Ophthalmol Vis Sci. 2013; 54(12):7362-7369.

97. Xu L, Blonska AM, Pumariega NM, et al. Reticular macular disease is associated with multilobular geographic atrophy in age-related macular degeneration. Retina. 2013;33(9):1850-1862.

98. Giocanti-Auregan A, Tadayoni R, Fajnkuchen F, Dourmad P, Magazzeni S, Cohen SY. Predictive value of outer retina en face OCT imaging for geographic atrophy progression. Invest Ophthalmol Vis Sci. 2015;56(13):8325-8330.

99. Schaal KB, Rosenfeld PJ, Gregori G, Yehoshua Z, Feuer WJ. Anatomic clinical trial endpoints for nonexudative age-related macular degeneration. Ophthalmology. 2016;123(5):1060-1079.

100. Arnold JJ, Quaranta M, Soubrane G, Sarks SH, Coscas G. Indocyanine green angiography of drusen. Am J Ophthalmol. 1997;124(3): 344-356.

101. Alten F, Clemens CR, Heiduschka P, Eter N. Localized reticular pseudodrusen and their topographic relation to choroidal watershed zones and changes in choroidal volumes. Invest Ophthalmol Vis Sci. 2013;54(5):3250-3257.
102. Vongkulsiri S, Ooto S, Mrejen S, Suzuki M, Spaide RF. The lack of concordance between subretinal drusenoid deposits and large choroidal blood vessels. Am J Ophthalmol. 2014;158(4):710-715.

103. Querques G, Kamami-Levy C, Blanco-Garavito R, et al. Appearance of medium-large drusen and reticular pseudodrusen on adaptive optics in age-related macular degeneration. Br J Ophthalmol. 2014; 98(11):1522-1527.

104. Zhang Y, Wang X, Rivero EB, et al. Photoreceptor perturbation around subretinal drusenoid deposits as revealed by adaptive optics scanning laser ophthalmoscopy. Am J Ophthalmol. 2014;158(3): 584-596.e1.

105. Meadway A, Wang X, Curcio CA, Zhang Y. Microstructure of subretinal drusenoid deposits revealed by adaptive optics imaging. Biomed Opt Express. 2014;5(3):713-727.

106. Zhang Y, Wang X, Godara P, et al. Dynamism of dot subretinal drusenoid deposits in age-related macular degeneration demonstrated with adaptive optics imaging. Retina. Epub 2017 Feb 10.

107. Mrejen S, Sato T, Curcio CA, Spaide RF. Assessing the cone photoreceptor mosaic in eyes with pseudodrusen and soft Drusen in vivo using adaptive optics imaging. Ophthalmology. 2014;121(2):545-551.

108. Flamendorf J, Agron E, Wong WT, et al. Impairments in dark adaptation are associated with age-related macular degeneration severity and reticular pseudodrusen. Ophthalmology. 2015;122(10): 2053-2062.

109. Sevilla MB, McGwin G Jr, Lad EM, et al. Relating retinal morphology and function in aging and early to intermediate age-related macular degeneration subjects. Am J Ophthalmol. 2016;165:65-77.

110. Steinberg JS, Fitzke FW, Fimmers R, Fleckenstein M, Holz FG, Schmitz-Valckenberg S. Scotopic and photopic microperimetry in patients with reticular drusen and age-related macular degeneration. JAMA Ophthalmol. 2015;133(6):690-697.

111. Neely D, Zarubina AV, Clark ME, et al. Association between visual function and subretinal drusenoid deposits in normal and early agerelated macular degeneration eyes. Retina. 2017;37(7):1329-1336.

112. Forte R, Cennamo G, de Crecchio G, Cennamo G. Microperimetry of subretinal drusenoid deposits. Ophthalmic Res. 2014;51(1):32-36.

113. Querques G, Massamba N, Srour M, Boulanger E, Georges A, Souied EH. Impact of reticular pseudodrusen on macular function. Retina. 2014;34(2):321-329.

114. Ooto S, Ellabban AA, Ueda-Arakawa N, et al. Reduction of retinal sensitivity in eyes with reticular pseudodrusen. Am J Ophthalmol. 2013; 156(6):1184-1191.e2.

115. Wu Z, Ayton LN, Makeyeva G, Guymer RH, Luu CD. Impact of reticular pseudodrusen on microperimetry and multifocal electroretinography in intermediate age-related macular degeneration. Invest Ophthalmol Vis Sci. 2015;56(3):2100-2106.

116. Alten F, Heiduschka P, Clemens CR, Eter N. Multifocal electroretinography in eyes with reticular pseudodrusen. Invest Ophthalmol Vis Sci. 2012;53(10):6263-6270.

117. Alten F, Heiduschka P, Clemens CR, Eter N. Longitudinal structure/ function analysis in reticular pseudodrusen. Invest Ophthalmol Vis Sci. 2014;55(9):6073-6081

118. Huisingh C, McGwin G Jr, Neely D, et al. The association between subretinal drusenoid deposits in older adults in normal macular health and incident age-related macular degeneration. Invest Ophthalmol Vis Sci. 2016;57(2):739-745.

119. Gil JQ, Marques JP, Hogg R, et al. Clinical features and long-term progression of reticular pseudodrusen in age-related macular degeneration: findings from a multicenter cohort. Eye (Lond). 2017;31(3): 364-371.

120. Finger RP, Wu Z, Luu CD, et al. Reticular pseudodrusen: a risk factor for geographic atrophy in fellow eyes of individuals with unilateral choroidal neovascularization. Ophthalmology. 2014;121(6): 1252-1256

121. Chang YS, Kim JH, Yoo SJ, Lew YJ, Kim J. Fellow-eye neovascularization in unilateral retinal angiomatous proliferation in a Korean population. Acta Ophthalmol. 2016;94(1):e49-e53. 
122. Sawa M, Ueno C, Gomi F, Nishida K. Incidence and characteristics of neovascularization in fellow eyes of Japanese patients with unilateral retinal angiomatous proliferation. Retina. 2014;34(4):761-767.

123. Ravera V, Bottoni F, Giani A, Cigada M, Staurenghi G. Retinal angiomatous proliferation diagnosis: a multiimaging approach. Retina. 2016;36(12):2274-2281.

124. Marsiglia M, Boddu S, Chen CY, et al. Correlation between neovascular lesion type and clinical characteristics of nonneovascular fellow eyes in patients with unilateral, neovascular age-related macular degeneration. Retina. 2015;35(5):966-974.

125. Naysan J, Jung JJ, Dansingani KK, Balaratnasingam C, Freund KB. Type 2 (Subretinal) neovascularization in age-related macular degeneration associated with pure reticular pseudodrusen phenotype. Retina. 2016;36(3):449-457.

126. Cho HJ, Yoo SG, Kim HS, et al. Risk factors for geographic atrophy after intravitreal ranibizumab injections for retinal angiomatous proliferation. Am J Ophthalmol. 2015;159(2):285-292.e1.

127. Munk MR, Ceklic L, Ebneter A, Huf W, Wolf S, Zinkernagel MS. Macular atrophy in patients with long-term anti-VEGF treatment for neovascular age-related macular degeneration. Acta Ophthalmol. 2016; 94(8):e757-e764.
128. Saito M, Iida T, Kano M, Itagaki K. Two-year results of combined intravitreal ranibizumab and photodynamic therapy for retinal angiomatous proliferation. Jpn J Ophthalmol. 2016;60(1):42-50.

129. Nghiem-Buffet S, Giocanti-Auregan A, Jung C, et al. Reticular pseudodrusen are not a predictive factor for the 1-year response to intravitreal ranibizumab in neovascular age-related macular degeneration. Retina. 2017;37(1):53-59.

130. Bandello F, Sacconi R, Querques L, Corbelli E, Cicinelli MV, Querques G. Recent advances in the management of dry age-related macular degeneration: a review. F1000Res. 2017;6:245.

131. Corvi F, Souied EH, Falfoul Y, et al. Pilot evaluation of short-term changes in macular pigment and retinal sensitivity in different phenotypes of early age-related macular degeneration after carotenoid supplementation. Br J Ophthalmol. 2017;101(6):770-773.
Clinical Ophthalmology

\section{Publish your work in this journal}

Clinical Ophthalmology is an international, peer-reviewed journal covering all subspecialties within ophthalmology. Key topics include: Optometry; Visual science; Pharmacology and drug therapy in eye diseases; Basic Sciences; Primary and Secondary eye care; Patient Safety and Quality of Care Improvements. This journal is indexed on

\section{Dovepress}

PubMed Central and CAS, and is the official journal of The Society of Clinical Ophthalmology (SCO). The manuscript management system is completely online and includes a very quick and fair peer-review system, which is all easy to use. Visit http://www.dovepress.com/ testimonials.php to read real quotes from published authors. 